\title{
Multi-Channel Experimental of Double-Layers Vibration Isolation Based On DSP
}

\author{
Zhengtao Yan ${ }^{\mathrm{a}, *}$, Jianguo Xue ${ }^{\mathrm{b}}$, Xuanyan Qian $^{\mathrm{c}}$ and Chi Huang ${ }^{\mathrm{d}}$ \\ Ship and ocean academy Naval Univ. of Engineering Wuhan 430033, China. \\ *, a piglad@vip.qq.com, b867566104@qq.com, c4799592@qq.com, d1436163504@qq.com
}

Keywords: $F_{x}$ LMS algorithm, dual-core controller of DSP+FPGA, vibration control.

\begin{abstract}
. based on the theoretical research of multi-channel active control, this article developed a four-channel dual-core controller based on DSP+FPGA, and achieved good control effects, through the four channels in double layer vibration isolation platform, under the test of LMS system. According to the problems about slow convergence of speed in control systems, large delay in calculating of DSP caused by large amount of calculation, and not good reference signal, which are argued thoroughly, solutions were offered.
\end{abstract}

\section{Introduction}

With the further study of vibration control theory and experiment, active control becomes an important branch of vibration control engineering. The traditional passive vibration isolation has obvious effect on the high frequency vibration, but it is not ideal for the isolation of low-frequency vibrations. The active control has the characteristics of "vibration remedy vibration" and automatic tracking vibration, which has better control effect on low-frequency vibration. In recent years, with the miniaturization of electronic devices and the rapid process of data processing, active control has become the focus of domestic and foreign experts [1-2]. The principle is that the vibration signal is collected by sensors, and then the actuator is used to generate a signal with the same frequency and amplitude as the vibration signal to counteract it [3]. The adaptive control can monitor the controlled object and the disturbance excitation in real time and update the control parameters in real time to keep the system performance index optimal. Adaptive control is divided into adaptive filter control, self-tuning control and so on. Among them, the adaptive filter control in the Vibration field applies more [4-5]. The most widely used is the Feedforward FxLMS (filtered-x least mean square) algorithm.

The DSP adopts the Harvard structure system, has the multi bus structure, and can carry on the pipeline operation. It's hardware multiplier and the efficient multiple and accumulate operation (MAC) are especially suitable for the digital filter signal processing computation. Due to the advantages of DSP, most of the main engineering multi-channel active controllers are based on their development. However, the DSP is the partial engineering hardware. When the control algorithm writes to the hardware from the simulation software, it often involves the program language conversion and the compilation question, so it is very inconvenient for the algorithm in the verification of the research stage.

\section{FxLMS Algorithm and Controller Hardware}

\subsection{Fxlms Algorithm}

In active vibration control, the application of standard LMS algorithm is likely to cause system instability, because the influence of components makes the control signal error channel, namely the secondary channel, resulting inequality of time between the signals of the reference sensor and the error sensor. There are two ways to solve this problem: one is to place the reciprocal $1 / \mathrm{S}(\mathrm{n})$ of the secondary channel transfer function $s(n)$ in the error feedback loop in tandem with $S(n)$, offset the effect of $S(n)$; the other is an estimate of $S(n)$ added between the reference sensor and the weight update of LMS algorithm to delay the reference signal and to achieve the equivalence of time. That 
is FxLMS algorithm. Because 1/S(n) may not exist, the first way cannot be implemented, so the FXLMS algorithm is a more efficient method.

Figure 1 is the FXLMS algorithm structure diagram, from which it can be seen that the influence of the secondary channel on the active control is considered. The $\mathrm{S}(\mathrm{z})$ behind the adaptive filter is the actual secondary channel, and $H_{p}(n)$ represents the primary channel transfer function, and the $\hat{S}(n)$ before the adaptive Control algorithm is the estimation of the secondary channel.

The error signal at $\mathrm{n}$ time is:

$$
\mathrm{e}(n)=d(n)-S(n)
$$

The control output quality of the FIR electric filter is

$$
u(n)=\sum_{t=0}^{N-1} W_{i}(n) x(n-i)=\vec{w}(n)^{T} \vec{x}(n)^{T}=\vec{x}(n)^{T} \vec{w}(n)^{T}
$$

Where $\mathrm{W}(\mathrm{n})$ is the Nx1 order weighted coefficient vector of the filter's $n$ point, $\mathrm{X}(\mathrm{n})$ is the Nx1 order reference signal vector.

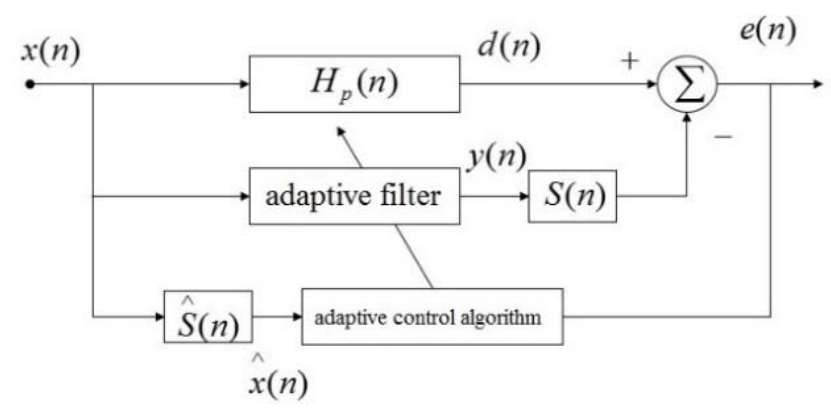

Fig. 1. Structure of FxLMS Algorithm

The control signal of the filter output is not the direct control quantity required, because it should consider the error channel between the error sensor and the controller.

The weight vector of the nth sampling point filter is:

$$
W(n)=\left[w_{0}, w_{1}, \ldots, w_{L-1}\right]^{T}
$$

If the estimation model of the error channel identification is a transverse filter with length $\mathrm{M}$, the weight vector is

$$
S(n)=\left[s_{0}, S_{1}, \ldots, s_{M-1}\right]^{T}
$$

The error channel $\mathrm{S}(\mathrm{z})$ is approximated by an estimated model, at which point the output of the $\hat{S}(n)$ error sensor is expressed as:

$$
\begin{aligned}
e(n) & =d(n)-S(n)^{T} y(n)=d(n)-\sum_{l=0}^{L-1} w_{l} \sum_{\mathrm{m}=0}^{\mathrm{M}-1} s_{m} x(n-m-l) \\
& =d(n)-\sum_{m=0}^{M-1} s_{m} \sum_{l=0}^{L-1} w_{l} x(n-l-m) \\
\hat{x}(n) & =\sum_{m=0}^{M-1} s_{m} x(n-m) e(n)=d(n)-\sum_{l=0}^{L-1} w_{l} \hat{x}(n-l)
\end{aligned}
$$

Input vectors for secondary channels are

$$
\hat{X}(n)=[\hat{x}(n), \hat{x}(n-1), \ldots, \hat{x}(n-L+1)]^{T}
$$


Then

$$
e(n)=d(n)-[\hat{X}(n)]^{T} W(n)
$$

By LMS algorithm, the iteration of weight vector changes in the steepest direction of negative gradient, so it can be expressed as

$$
W(n+1)=W(n)-2 \lambda e(n) \hat{x}(n)
$$

$\lambda$ is the iterated long of the weight vector in the FxLMS algorithm. The weight vector is used to approximate the optimal solution by recursion formula, when the error signal satisfies the performance function criterion, the ideal control effect can be achieved.

\subsection{Controller Hardware}

The active control system has the characteristics of fast response time, high sampling frequency, multi-channel parallel control and large computational load, which requires the controller hardware to have high data processing ability. In order to ensure the processing speed of complex data, the system adopts FPGA+DSP dual-core processing architecture. The FPGA is responsible for the signal acquisition and output, and DSP is responsible for digital filtering, algorithm operation and communication with the host computer [6-9].

FPGA operation speed cannot be affected by the number of external devices. Because the management of each external device of FPGA is independent, its code execution is parallel to meet the requirements of high speed acquisition and output. However, it is difficult to realize the algorithm, and the operation speed is not high. DSP makes up for the weakness of FPGA and can be more flexible and convenient to write control algorithm through the $\mathrm{C}$ or $\mathrm{C}++$ language programming. This control system uses the XC6SLX16 chip in the Spartan-6 series produced by Xilinx company as signal acquisition chip, and DSP Chip uses TMS320C6748[6-9].

In signal acquisition and output, the ADC (analog Digital Converter) used in the system needs the function of high-speed, high resolution, bipolar input and multi-channel signal conditioning, by using ADI's AD7606 chip. Digital-analog converters need to meet the requirements of high speed, high resolution, bipolar output and multi-channel, by using ADI's AD5724, which has four-channel parallel outputs, single polarity and bipolar as well as adjustable voltage range output.

In the communication between the host computer and the slave computer, because of the large amount of data transmitted by the control system, the requirement for the stability of the communication interface is high, and the Ethernet is used for data interaction with the host computer. In the case of limited hardware conditions, in order to ensure the calculation of the control algorithm, DPS and host computer communication use external chip [10-11].

\section{Active Vibration Isolation Experiment}

\subsection{Platform Construction}

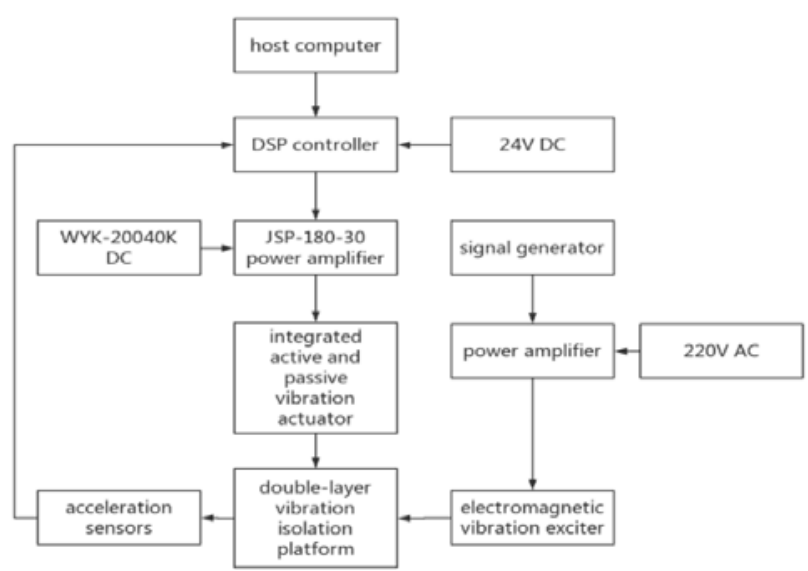

Fig.2. Main Hardware Flow Chart of Test 


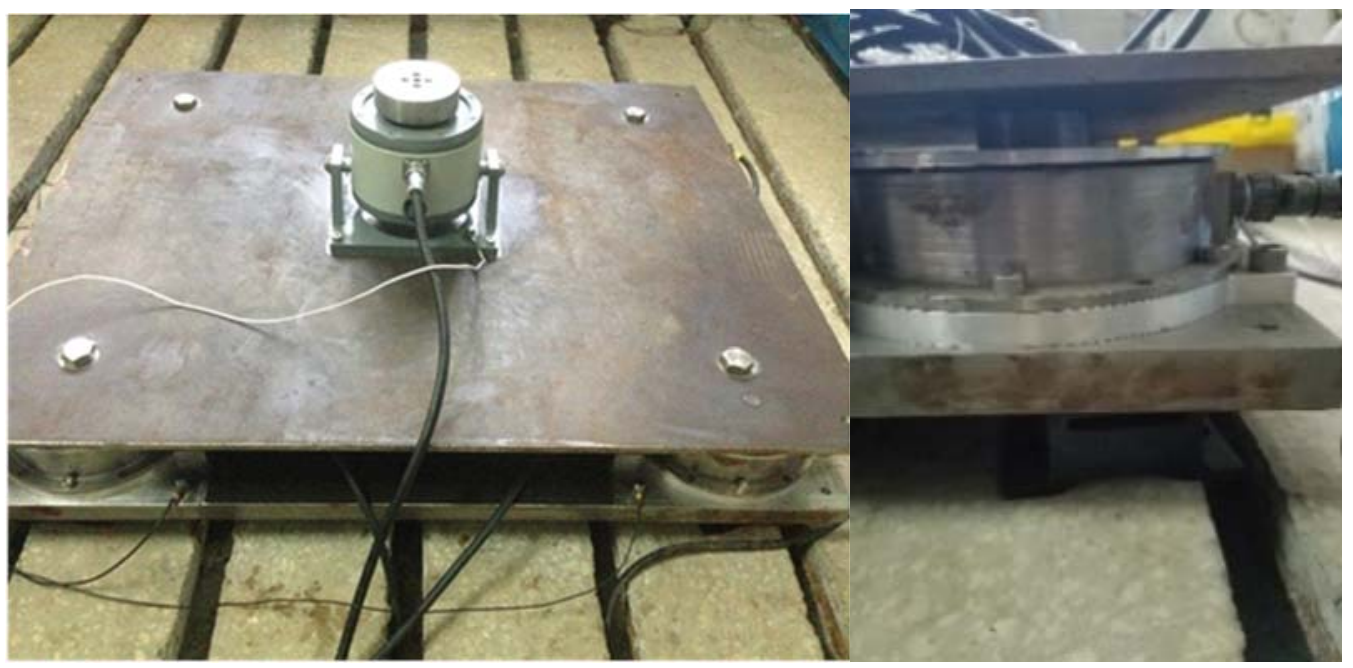

Fig.3. Material Diagram and Side View of Double Deck Vibration Isolation Platform

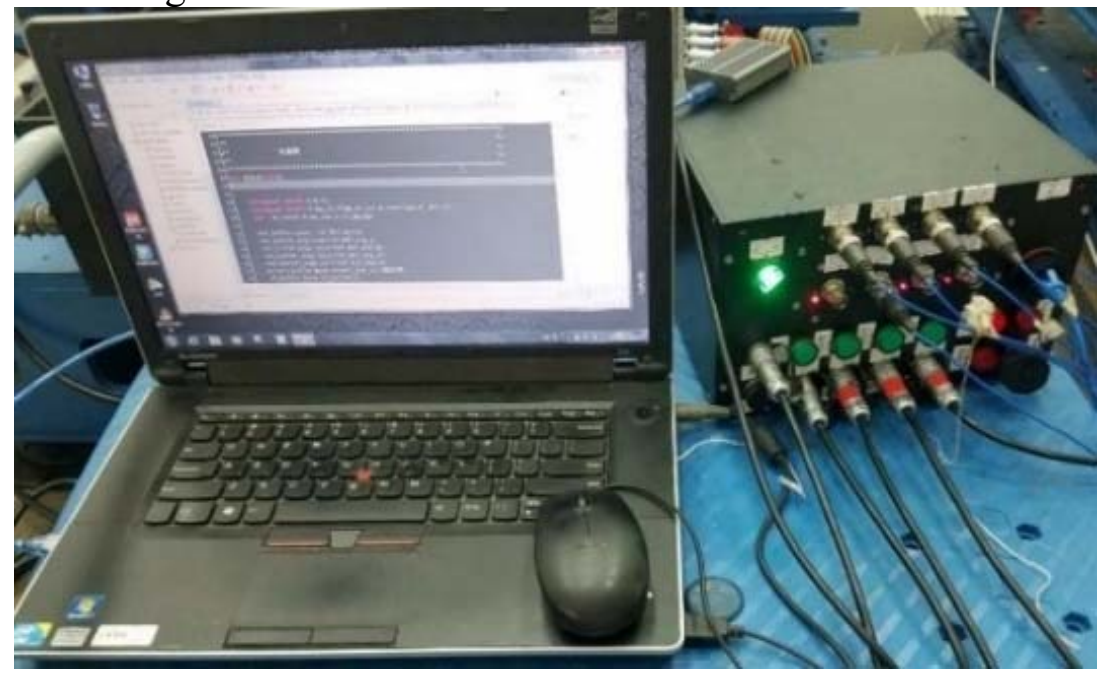

Fig. 4. Controller in Operation

Fig. 3 shows the two-layer vibration isolation platform, and the acceleration signal of the middlelevel vibration isolator is used as the evaluation index. The middle of the upper plate is an electromagnetic exciter, and the upper plate and the lower plate are equipped with four independent developed passive-negative integrated vibration isolators, as the executing units of active control. The whole system uses 5 accelerometer sensors, which are arranged at the bottom of the exciter and at the bottom of the four mixed isolators to collect the reference signal and the error signal.

\subsection{Test Related Problems and Solutions}

Reference Signal Processing

In the experiment, in addition to the complex similar white noise, the reference signal also has a large value step signal, because of the instability of the excitation source, the working frequency of the vibration source will fluctuate to a certain extent in the set working frequency, which affects the control effect of the whole system and makes the system prone to divergence.

The frequency of the sampled reference signal is approximately $f$, and the band-pass filter is used to filter the signal. The bandwidth can be set to $(F \pm n) H z, n$ value is determined by the amplitude fluctuation of the source frequency, which ensures that the reference signal does not exceed the bandpass filter when it fluctuates [12]. Similarly, according to the normal range of the reference signal, a magnitude limit is given to the doped large value step signal, so the signal exceeding the specified threshold will be cut off.

Fast Convergence of Multichannel Control

In order to ensure the convergence of each control channel to obtain the overall control effect, adjusting the output gain and iteration step of the controller is a feasible operation, which can avoid the control divergence caused by channel crosstalk. This results in a very long adjustment time for the system to achieve full convergence. 
According to $W(n)=\left[w_{0}, w_{1}, \ldots, w_{L-1}\right]^{T}$ the weighted coefficients of the controllers participating in the iterative operation are a series of arrays. In the adaptive process, it is based on the formula $W(n+1)=W(n)+2 u X(n) e(n)$ to do continuous iterative computation. The initial value of $\mathrm{W}(\mathrm{n})$ can be set toW $(n)_{0}$, which will be $W(n)_{s}$ after convergence. When the vibration of the control target is stable enough, the iteration of $W(n)$ is still in progress after the control algorithm is finally convergent, but because $\mathrm{e}(\mathrm{n})$ is very small, theW $(\mathrm{n})_{s}$ is stabilized and vibrates in a small range of stable values. While the normal iterative, process $\mathrm{W}(\mathrm{n})_{0}$ starts with 0 , in order to reduce the iterative time, the convergent weight coefficient $\mathrm{W}(\mathrm{n})_{s}$ is saved and set to the initial value $\mathrm{W}(\mathrm{n})_{s}$ at the next control. In this way, the calculation iterations of $W(n)$ begins with the addition of close to the final stable value, eliminating the iterative process from the 0 to the stable value, so that the convergence speed of the algorithm is greatly improved.

Estimation of Dsp Chip Calculation and Its Improvement Method

In the selection of DSP chips, the computing speed (instruction cycle and MAC), the accuracy of operation and the completeness and convenience of development tools are the main problems. In the active vibration control, the operation precision and power dissipation of DSP can meet the requirements, so the calculation speed is the core problem.

For example, the MFXLMS algorithm is used in multi-channel active control system, in which there are $J$ road reference signal, $M$ road error signal and $K$ secondary channels, and the length of controller and secondary channel modeling filter is $L$ and $L_{s}$ respectively, then the multiplier number of the algorithm is $\left(\mathrm{L}+L_{S}\right) \times J \times M \times K$. For example, the vibration of a vibration isolation platform uses 1 reference signals, 4 error sensors and 4 secondary sources. The length of the controller and the secondary channel identification length are 128 , and the system sampling frequency is $1 \mathrm{KHz}$. In order to complete the arithmetic, the multiplication and accumulation of DSP chip cannot lower than $4 \times 10^{\wedge} 6$. Therefore, before the experiment, we can deduce the approximate calculation quantity according to the calculation and select the appropriate DSP chip.

Selecting the DSP chip with the highest operation speed can reduce the requirement of the control system to a large extent. But in practice, it often faced with the problem of insufficient computational capacity, so reducing the amount of computing from the software is the best choice.

(1) It can be seen from the FxLMS formula that reducing the orders of the controller and the secondary channel and the sampling frequency will reduce the calculation amount, but also lead to the poor control effect. Therefore, it can be adjusted according to the actual situation, reducing to ensure real-time operation appropriately.

(2) Cong the formula of coefficient iteration, the sampling signal is real-time calculation. If adopt multiple points, such as $\mathrm{n}$ points, and then bring into the calculation, it can make the calculation of the original $1 / \mathrm{n}$. At the same time, the output frequency of the controller is changed to the original $1 / \mathrm{n}$, and the real-time performance of the controller is reduced. In the active control, the vibration frequency of the control target is often low, and the output frequency of the controller is much higher than the vibration frequency of the control target, so the effectiveness of the control is still ensured.

In the experiment, digital filter, band-pass filter and signal threshold are calculated by using the internal code of DSP. If the external filter is used, the calculation quantity is further compressed, and more calculations are used in the control to improve the control effect.

\subsection{The Decentralized Four-Channel Active Vibration Isolation Test}

For the Double-layer vibration isolation platform, the decentralized four-channel active vibration isolation test is carried out with the excitation source of sine signal in $37 \mathrm{~Hz}$, the order of control and identification filter at 300, and the control iteration step at 5e-5. Results are showed in Figure 5 and Figue6: 


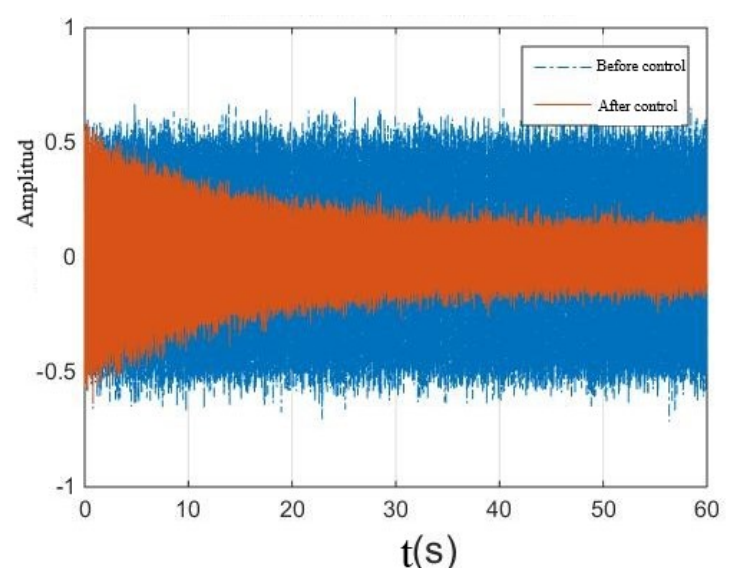

(a). The Control Effect of No.1

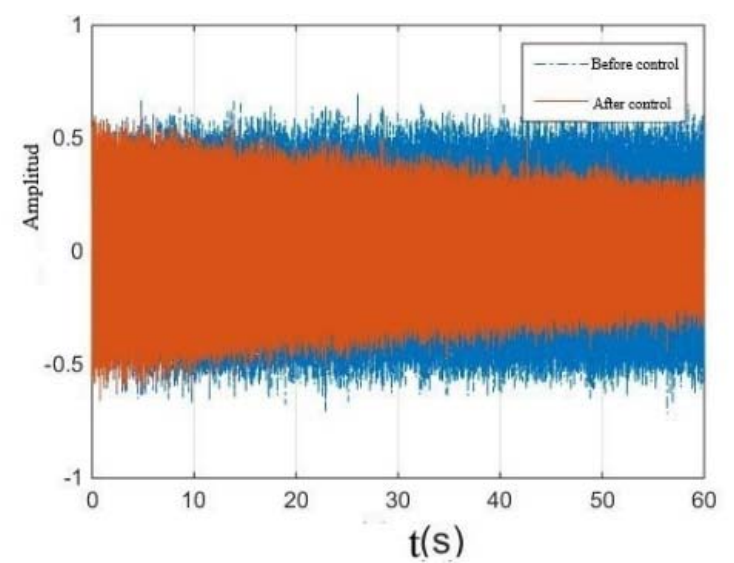

(c). The Control Effect of No.3

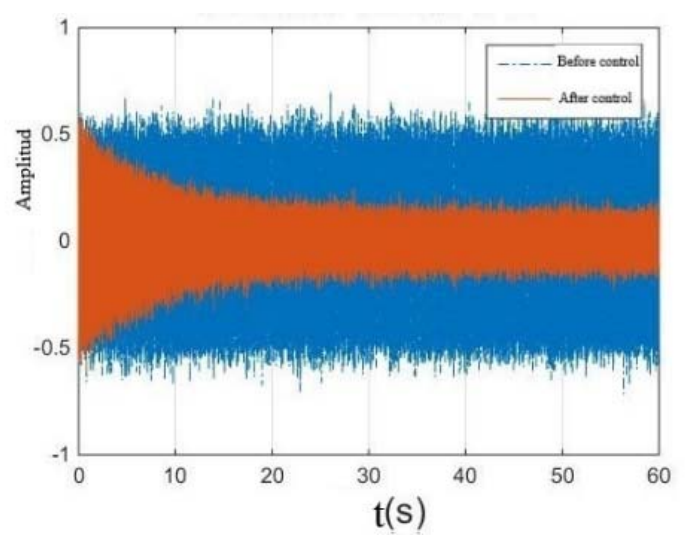

(b). The Control Effect of No.2

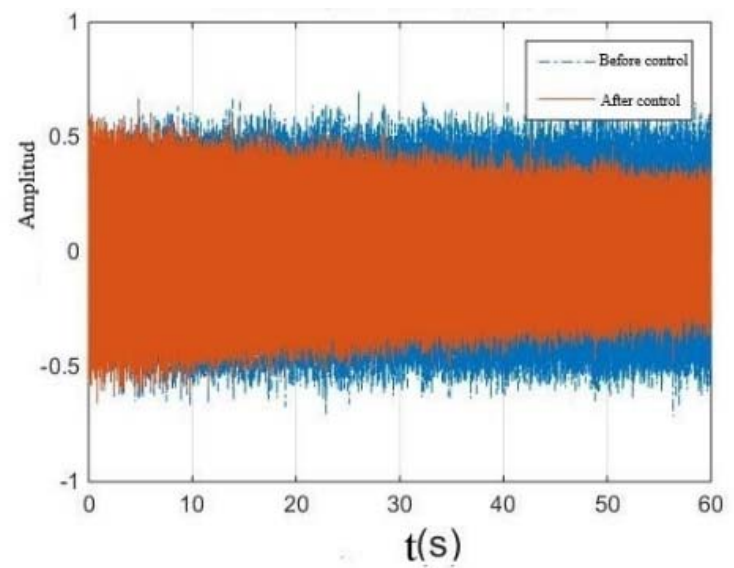

(d). The Control Effect of No.4

Fig.5. Multichannel Control Effect Without Fast Convergence Method

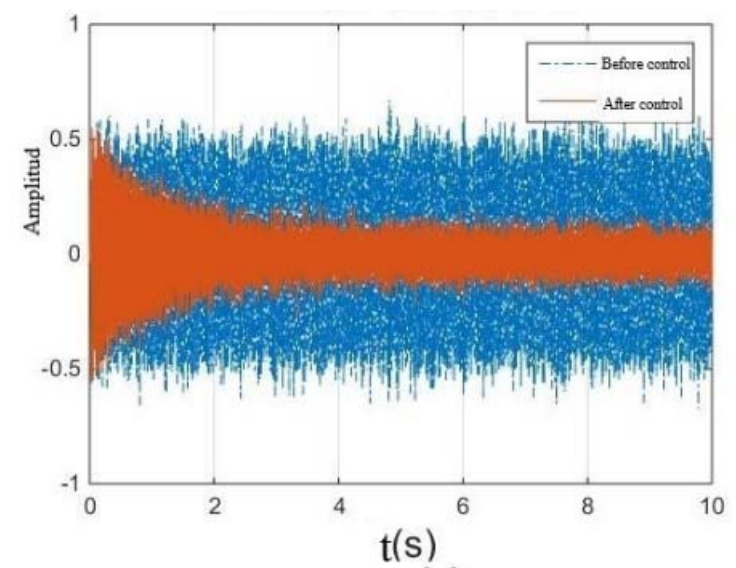

(a). The Control Effect of No.1

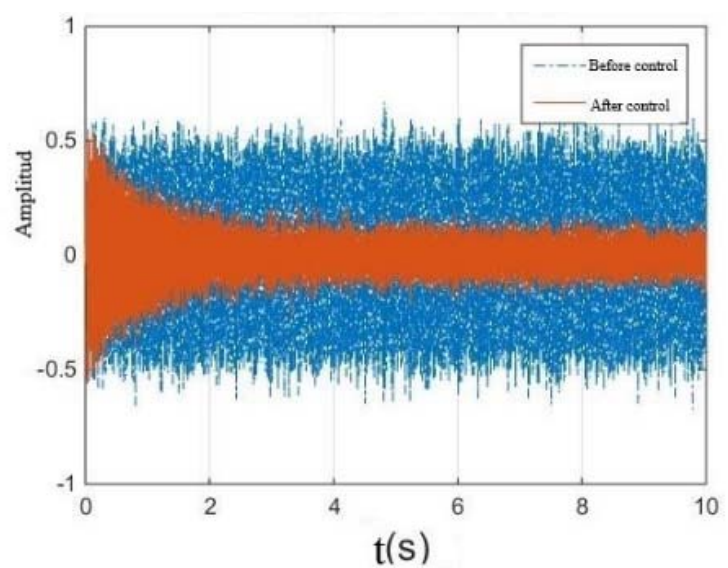

(b). The Control Effect of No.2 


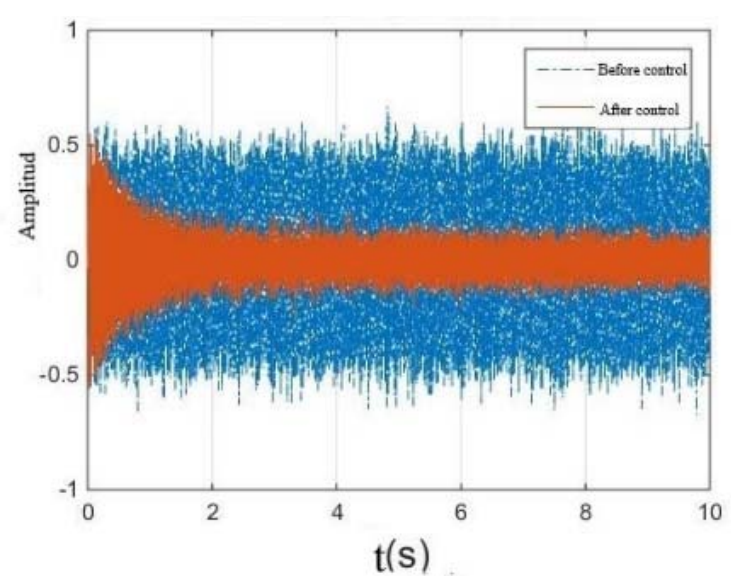

(c). The Control Effect of No.3

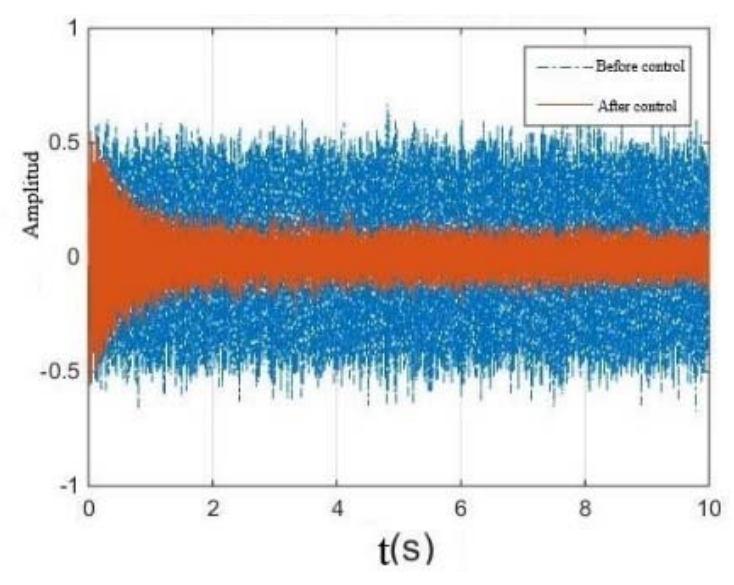

(d). The Control Effect of No.4

Fig. 6. Multichannel Control with Fast Convergence Method

From the experimental results, it can obviously improve the rate of convergence in control with the method of rapid convergence applied. In Fig.5, it takes a long time for iterative adaptive computation to obtain the optimal control effect for multi-channel control without using the fast convergence method. The control of No.1 and No.2 is nearly stable around 60s, while the iterative calculation of No. 3 and No.4 is still in progress. However, the convergence speed of control in four points gets improved remarkably, after applying the fast convergence method that was mentioned in chapter "Fast convergence of multichannel control", like Fig.6.

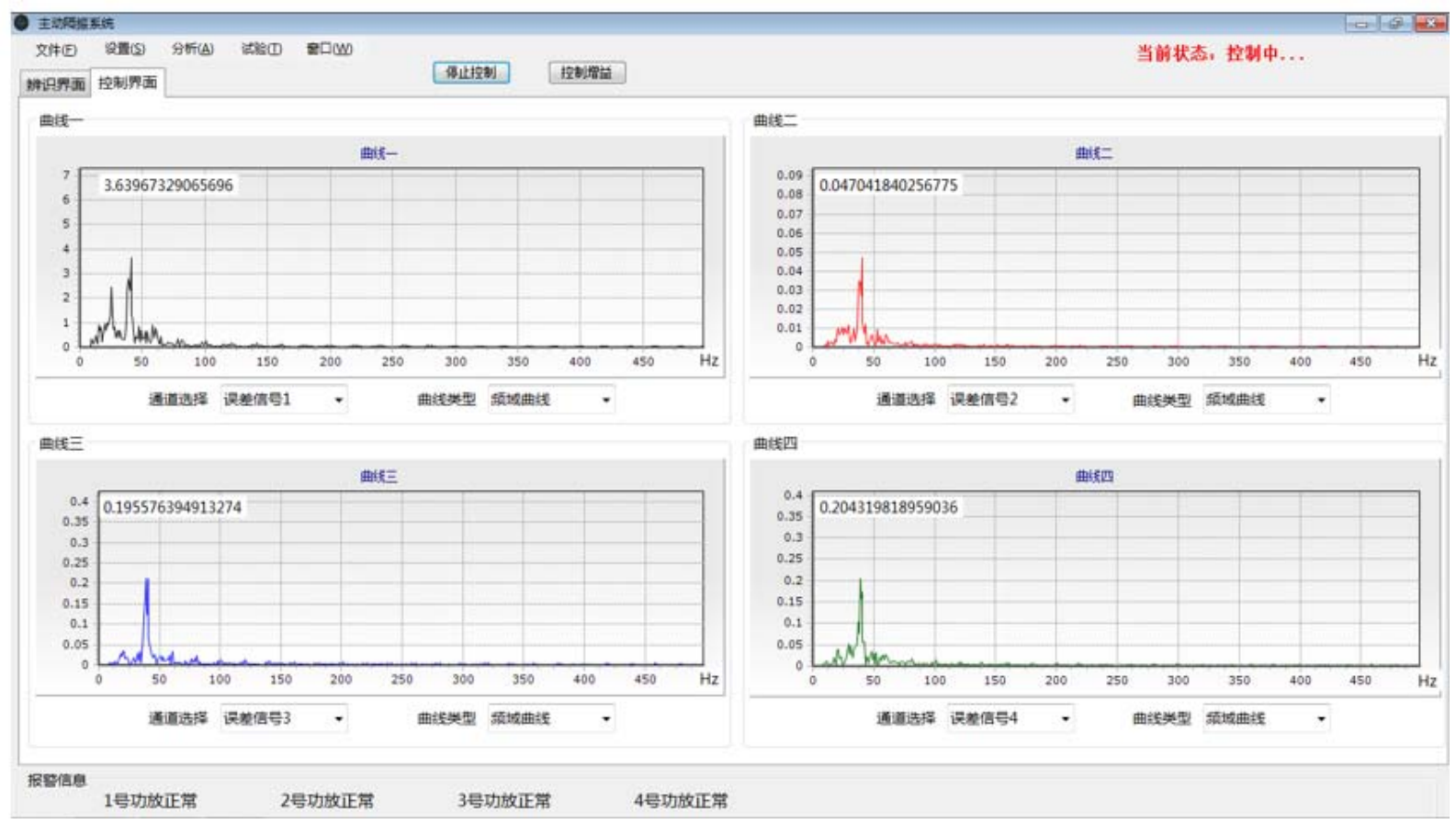

Fig. 7. Frequency Domain Curve for Controlling the Vibration of the First Four Points 


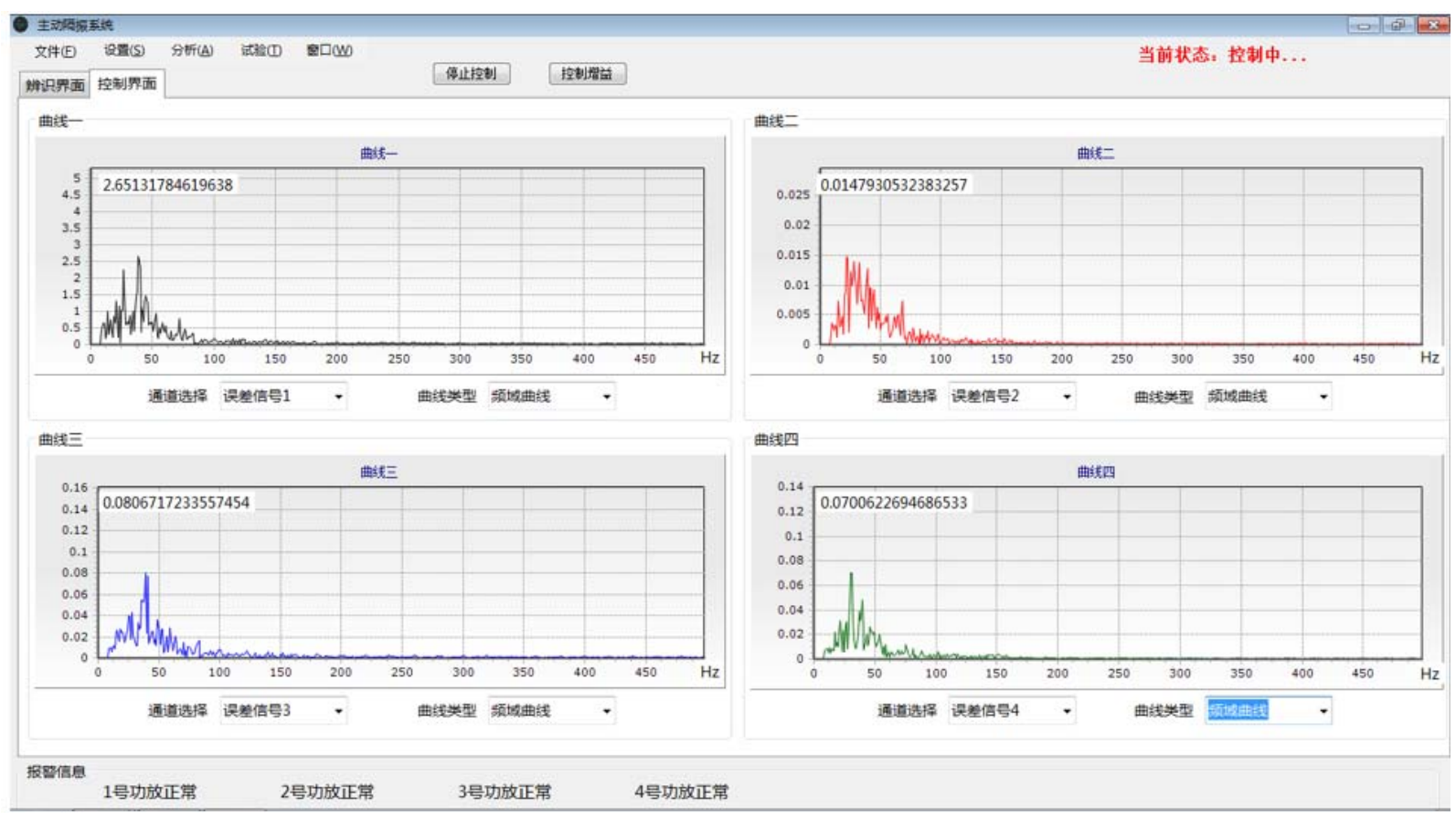

Fig. 8. Frequency Domain Curve of Vibration after Four Points of Control

As Fig. 7 shows, there are many spectral lines in the spectrum absorbed by the sensor, except of the given excitation source of $37 \mathrm{~Hz}$. There are two main reasons, one about structural resonance and the other is environmental noise. The existence of these lines brings difficulties in controlling, so some resemble methods need to be taken into the experiments. In Fig.7 and Fig.8, the collected error signals are processed by bandpass filtering between $20 \mathrm{~Hz}-60 \mathrm{~Hz}$, and the maximum amplitude is limited to prevent the interference of pulse signals. If it is necessary to further improve the signal quality and reduce the interference line spectrum of clutter near $37 \mathrm{~Hz}$, the bandwidth of bandpass filter can be reduced and more clutter will be filtered.
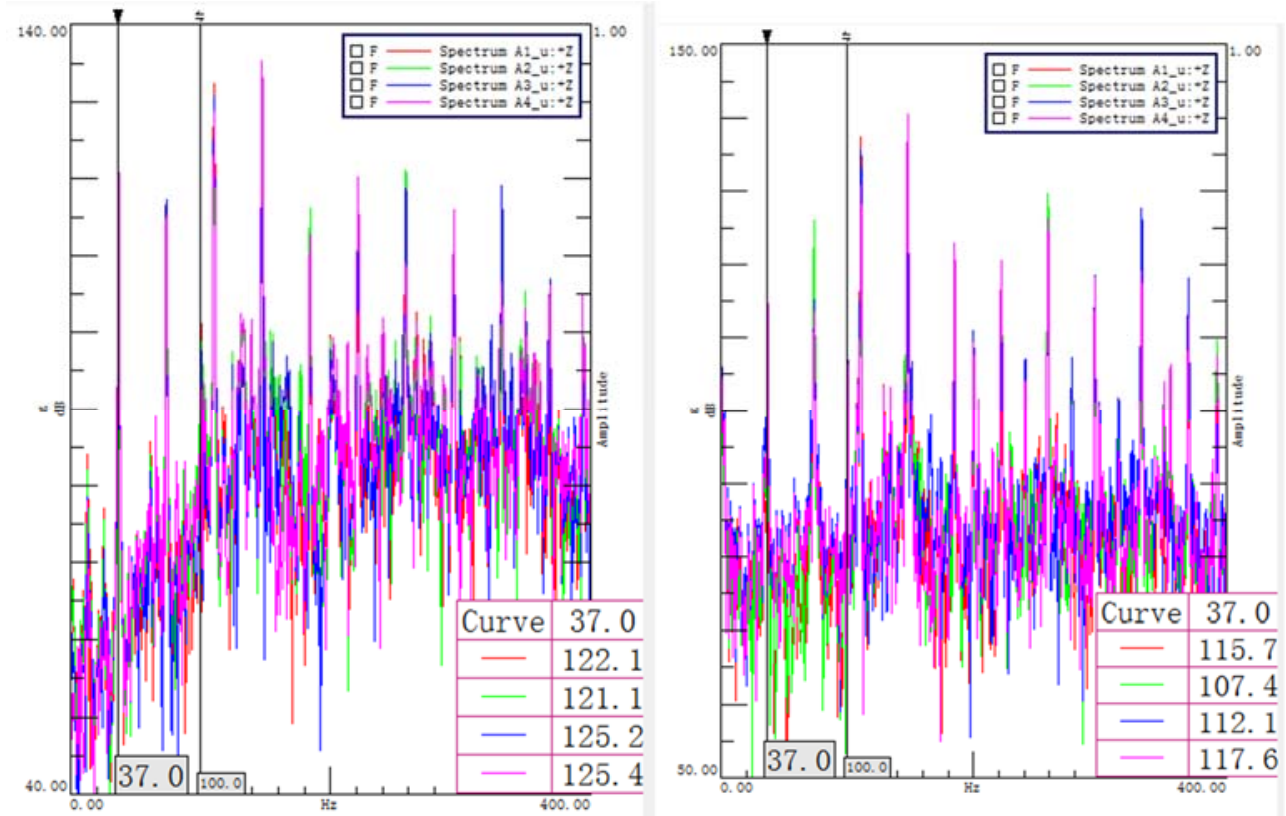

Fig. 9. Vibration and Noise at Different Points before and after Control

As it is shown in the frequency-domain curves of the vibration states of each point before and after control, the processing of the reference signal can obviously improve the accuracy of the signal and prevent the control instability caused by the pulse signal, from Fig.7 and Fig.8. Finally, LMS device was applied to detect the control target, in order to ensure the accuracy of the control effect. And as the results are shown in Fig. 9, the control effects of $7 \mathrm{db}, 14 \mathrm{db}, 13 \mathrm{db}$ and $8 \mathrm{db}$ are obtained by controlling four channels respectively. 


\section{Results and Discussion}

1. It can obviously improve the accuracy of the acquisition signal and prevent the instability of the control system caused by the pulse signal, in the methods of the reference signal with bandpass filter and amplitude limit.

2. The convergence rate of control is improved obviously by the iterative processing of weight coefficients.

3. It can reduce the computational amount of control algorithm and improve the real-time performance of the control system, with the calculation and improvement of DSP chip and development of DSP-FPGA dual-core controller.

4. The control effect of four-channel mean at $11 \mathrm{~dB}$ is obtained with the sine excitation signal of $37 \mathrm{~Hz}$, based on the Double-layer vibration isolation platform.

\section{References}

[1]. Thayer D, Campbell M, Vagners J, et al. Six-axis vibration isolation system using soft actuators and multiple sensor. Journal of Spacecraft and Rockets, 2002, Vol. 39(2): 206 212.

[2]. Eric H Anderson, Bowie Houghton. Elite-3 active vibration isolation workstation. Paper 433223, SPIE Smart Structures and Materials 2001: Industrial and Commercial Applications of Smart Structures Technologies.

[3]. C.R. Fuller, S.J. Elliott, P.A. Nelson. Active Control of vibration [M]. London: Academic Press, 1997.

[4]. Gao Liping. Research progress of active vibration control technology [J]. Modern machinery. 2005 (2); 35-39.

[5]. Gao Weipeng, He Qiwei, Yan Zhengtao. A double-layer vibration isolation test Device using FXLMS algorithm [J]. Noise and vibration control, 2016, 36 (40:70-75.

[6]. Diao Lijun, Dong Kan, Zhao Leiting, et. Electric traction control system of urban rail train based on dual-DSP-FPGA architecture [J]. Journal of Electrotechnical Technology, 2014,29 (01): 174180.

[7]. Peng Yu, Jiang Honglan, Yang Zhiming, et. Design of universal digital signal processing system based on DSP and FPGA [J]. Foreign electronic Measurement Technology, 2013, 32 (01): 1.

[8]. Yang Haigang, Sun Jiabin, Wang Wei. Review of development of FPGA device design technology [J]. Journal of Electronics and Information, 2010, 32 (03): 714-727.

[9]. Zhang Song, Li Yun. Modular design method of FPGA [J]. Journal of Electronic Measurement and instrumentation, 2014, 28 (05): 560-565.

[10]. Wu Kejia. Research and development of electro-hydraulic servo experimental bench controller based on Ethernet communication [D]. Harbin: Harbin University of Technology, 2015.

[11]. Lu Yang, Fu Bin, You Jiang. The application of W5100 to Ethernet communication in single chip microcomputer [J]. Heilongjiang Science and technology information, 2011, (05): 76+15.

[12]. H.J. Jeon, T.G. Chang, S. Yu. Et.al. A narrowband active noise control system with frequency corrector [J]. IEEE Transactions on Audio, Speech, and Language Processing, 2011, 19(4):9901002. 